\title{
De l'amateurisme en géologie: la réception française de la théorie de la dérive des continents (1920-1950)
}

Philippe Le Vigouroux, Gabriel Gohau

\section{Summary}

At the beginning of the $\mathrm{XX}^{\text {th }}$ century, Wegener proposed a theory - that of the roaming drift of the continents - unifying the rival theories of the Europeans and the Americans. As the work of a non-specialist who didn't trouble himself with specific details, it raised numerous criticisms from specialists in various disciplines though others welcomed and supported it. Some even understood that despite its flaws, it started a new research program.

Paradoxically, as regards its simplicity, nonspecialists - engineers, popularizers, secondary school teachers and even believers in para-sciences - gave it a favorable reception. Being amateurs, they continued to endorse it when specialists abandoned it.

Keywords: Continental Drift, Wegener, Research Program, Amateurs, Teaching, Popularization, Para-Sciences

Philippe Le Vigouroux, Gabriel Gohau, Centre François Viète d'épistemologie et d'histoire des sciences et des techniques, UFR Sciences et Techniques, 2, rue de la Houssinière, F-44322 Nantes Cedex 3 (ph.levigouroux@gmail.com) 


\section{Résumé}

Au début du $\mathrm{XX}^{\mathrm{e}}$ siècle, Wegener propose la théorie d'une dérive vagabonde des continents unissant les théories concurrentes des Européens et des Américains. Elle est l'œuvre d'un non spécialiste qui ne s'embarrasse pas de détails. C'est pourquoi elle provoque de nombreuses critiques chez les spécialistes des diverses disciplines. Certains cependant l'accueillent favorablement. Quelques-uns, même, comprennent que sous son aspect apparemment superficiel, elle amorce un nouveau programme de recherche.

Paradoxalement, dans la mesure de sa simplicité, elle reçoit un accueil favorable de non spécialistes: ingénieurs, vulgarisateurs, enseignants du secondaire et même de partisans des para-sciences. Tous amateurs, ils la feront vivre quand les spécialistes l'auront abandonnée.

En 1915, Alfred Wegener (1880-1930) publiait la première édition de son ouvrage Die Entstehung der Kontinente und Ozeane. ${ }^{1}$ Louvrage expose une théorie qu'il avait formulée en 1912 proposant une nouvelle conception du globe terrestre: l'auteur suppose que les continents (qu'il nomme «sial»), flottant sur des couches sous-jacentes, plus fluides et plus denses (le «sima»), sont mobiles à la surface du globe. Autrefois réunies en un continent unique, la Pangée, ces masses continentales se sont séparées par le jeu de gigantesques fractures qui deviennent des océans, les continents allant, ensuite, à la dérive jusqu'à occuper leur position actuelle.

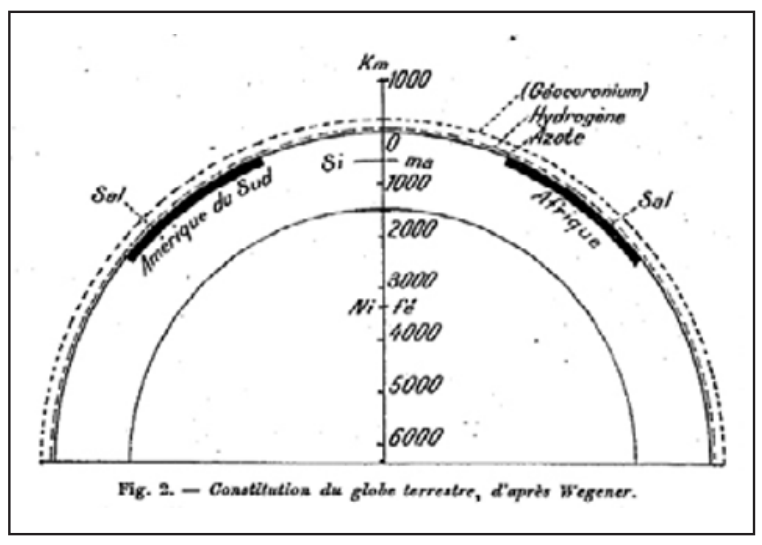

Gegnebin, Élie, 1922. La dérive des continents selon la théorie d'Alfred Wegener. Revue générale des sciences pures et appliquées, 33 (10):293-304 (p.296).

Représentation de la constitution du globe terrestre selon Wegener. Les continents de $S a l$ (ou Sial) flottent sur une couche de Sima, laissant apparaître celui-ci au fond des océans.

1 Wegener 1915. Ni cette édition, ni la suivante en 1920, ne seront traduites en français. 
Alfred Wegener, né à Berlin, fait des études de mathématique et de sciences naturelles dans diverses universités allemandes. En 1904, il soutient une thèse sur les Tables alphonsines, ${ }^{2}$ dirigé par l'astronome Julius Bauschinger, puis il rejoint son frère Kurt Wegener l'année suivante, à l'Institut aéronautique de Lindenburg où ils travaillent sur les ballons météorologiques qui permettent d'étudier la haute atmosphère. En 1906, il est invité en tant que météorologiste à rejoindre l'expédition Danmark conduite par Ludvig Mylius-Erichsen. Malgré la mort tragique de celui-ci en 1907, l'expédition se poursuit sur les deux années suivantes, comme prévu. À son retour en 1909, Wegener trouve un travail à l'Université de Marburg où il donne des cours d'astronomie, de physique et d'optique de l'atmosphère. Il publie, en 1911, Thermodynamik der Atmosphäre, ${ }^{3}$ un ouvrage qui devient une référence dans le domaine de la physique atmosphérique, ${ }^{4}$ puis participe à l'expédition danoise du capitaine Johan Peter Koch au Groenland entre 1912 et 1913. Mobilisé sur le front de l'Ouest, il est blessé à deux reprises dès 1914 et met à profit une longue convalescence pour reprendre ses recherches et ses écrits sur la dérive continentale. Reprenant le service actif, il est muté au Service météorologique de l'Armée, en Bulgarie, avant de revenir à l'Université de Marburg, en 1918, toujours comme météorologiste.

Spécialiste de la physique de l'atmosphère, Wegener n'est pas un spécialiste de tectonique ou de physique du globe, disciplines apparues au début du XIX ${ }^{\mathrm{e}}$ siècle dont le champ s'est progressivement doté d'outils de formalisation $^{5}$ qui le rendent difficilement accessible aux amateurs pourtant nombreux en géologie. ${ }^{6}$ Cependant, paradoxalement, du fait de son échelle globale et de son assise pluridisciplinaire, la tectonique devient accessible à des spécialistes d'autres disciplines, voire à des «amateurs». Alfred Wegener n'est pas au sens propre un «amateur». Pourtant, ce spécialiste d'une autre discipline, la géophysique de l'atmosphère, entraînera dans son sillage une cohorte d'amateurs de toutes sortes.

Émise en 1912, sa théorie devient accessible en France dix ans plus tard. En 1922, elle s'offre aux lecteurs français, par le biais d'un article d'Élie $^{7}$

2 Wegener 1905.

3 Wegener 1911.

4 McCoy 2006, 10.

5 Cette évolution est illustrée, par exemple, par Léonce Élie de Beaumont (1798-1874), géologue et secrétaire perpétuel de l'Académie des sciences. Dans un mémoire présenté en 1829 à l'Académie des sciences, il amorce un scénario qu'il formalisera grâce aux outils de la géométrie, qui explique la formation des montagnes selon des forces réparties en un réseau pentagonal. Élie de Beaumont 1829-1830.

6 Porter 1973.

7 Gagnebin 1922. Sur le contexte de l'après-guerre et la préparation du Congrès géologique international de Bruxelles de 1922, voir Le Vigouroux 2011. 
Gagnebin (1891-1949), assistant de Maurice Lugeon au laboratoire de géologie de l'Université de Lausanne, qui en fait une présentation détaillée dans la Revue générale des sciences pures et appliquées. Les débats inaugurés par ce texte puis la traduction en français ${ }^{8}$ de la troisième édition du livre de Wegener par Manfred Reichel, dans le laboratoire de géologie de l'Université de Neuchâtel, ${ }^{9}$ seront largement médiatisés au cours des années suivantes.

Le présent article se concentre sur la réception de la théorie d'Alfred Wegener par les géologues français, puis sur sa diffusion dans un public plus large, constitué d'amateurs, souvent concepteurs de théories concurrentes, et d'enseignants. Notre propos sera limité au quart de siècle qui suit les débats initiaux, période au cours de laquelle, la théorie ne trouve plus guère de soutien chez les spécialistes académiques, avant de susciter un nouvel intérêt dans les milieux universitaires quand de nouveaux résultats sont obtenus dans le domaine du paléomagnétisme.

\section{Jugements de spécialistes sur une synthèse d'un nouveau type}

$\mathrm{Au}$ moment où Wegener aborde la question, deux théories principales s'opposent pour expliquer la conformation de la surface terrestre. La première, qui prédomine en Europe, est celle de l'Autrichien Eduard Suess ${ }^{10}$ (1831-1914), complétée par les apports de Marcel Bertrand et Pierre Termier sur les nappes de charriage ${ }^{11}$ et d'Émile Haug sur les géosynclinaux..$^{12}$ Le globe terrestre, en refroidissant, se contracte, provoquant des effondrements successifs de l'écorce. Une portion de la surface terrestre peut devenir successivement un domaine océanique ou un domaine continental: l'écorce ne présente pas de différence de nature entre ces deux domaines. Les chaînes de montagnes surgissent au niveau de fosses océaniques où se sont accumulés des sédiments, sous l'effet de compressions entre deux masses continentales.

La seconde, qui a la faveur des scientifiques américains, est celle de l'isostasie et de la permanence des traits de la Terre, défendue par le géologue Bailey Willis ${ }^{13}$ (1857-1949). Elle repose sur la distinction entre deux domaines de composition et de propriétés différentes, l'un continental, l'autre océa-

8 Wegener 1924. Il s'agit d'une traduction de la troisième édition allemande de l'ouvrage de Wegener, publiée en 1922: Die Entstehung der Kontinente und Ozeane, F. Vieweg, Braunschweig, $144 \mathrm{p}$.

9 Schaer 1991.

10 Suess 1885-1909.

11 Bertrand 1887 et Termier 1907.

12 Haug 1900, 1907.

13 Willis 1910. 
nique constitué de roches plus denses que le précédent. Les grands bassins océaniques sont des structures permanentes de la surface de la Terre dont l'emplacement ne s'est jamais modifié et dont les contours connaissent de faibles changements.

Loin de rejeter ces théories, Wegener propose de les dépasser en en faisant une synthèse. Lui-même écrit en 1928, dans la quatrième et dernière édition de son texte:

Si nous prenons comme base la théorie des translations, nous répondons à toutes les exigences justifiées, tant celles de la loi des anciennes liaisons continentales qu'à celles de la permanence. Nous n'avons qu'à énoncer ces lois comme il suit: Ponts continentaux? Oui, non pas grâce à des continents intermédiaires affaissés, mais à des socles continentaux jadis contigus. Permanence? Oui, pas de chaque continent ou océan pris individuellement, mais permanence de la surface océanique totale et de la surface continentale totale prise en bloc. ${ }^{14}$

Sa synthèse dépasse aussi les frontières des champs disciplinaires. En effet, s'il cite de nombreux auteurs, ce n'est pas précisément, comme l'avaient fait ses prédécesseurs, à une synthèse des études des géologues de terrain que se livre Wegener. Pour défendre son «hypothèse de travail», il se fonde sur des données scientifiques variées. Dans son ouvrage en 1922, il énumère dans une partie intitulée «Les preuves», tous les arguments utiles qu'il a puisés dans différents domaines scientifiques: arguments tirés de la géophysique qui le conduisent à distinguer des soubassements continentaux et océaniques de natures différentes; arguments géologiques qui montrent une continuité des terrains et des structures d'une rive à l'autre des océans; arguments paléontologiques et biologiques montrant une continuité des espèces actuelles et fossiles retrouvées sur les deux rives d'un océan; arguments paléoclimatiques comme la répartition des gisements de charbon ou les traces de glaciations anciennes; arguments géodésiques, enfin, qui permettent de supposer et d'évaluer un déplacement des continents en comparant les mesures de latitudes récoltées au cours des cinquante années précédentes.

Wegener construit sa synthèse sans être en mesure d'évaluer la validité des données sur lesquelles il s'appuie et en les simplifiant souvent. L'éventail des données utilisées couvre en effet des disciplines scientifiques différentes qu'aucun spécialiste ne pourrait toutes ensemble maîtriser. Maurice Gignoux (1881-1955), professeur de géologie à la faculté de Strasbourg, le constate en 1925: «Le principal mérite de Wegener a été de nous apporter, précisément à ce moment, [...] beaucoup d'idées..$^{15}$ De son point de vue, seul un nongéologue pouvait avoir le recul nécessaire pour le faire, en simplifiant les

14 Wegener 1937, 21.

15 Gignoux 1925, 140. 
problèmes qui se posaient et en ne s'embarrassant pas d'un vocabulaire que Gignoux qualifie de «brumeux»:

D'abord Wegener n'est point un géologue, mais un géophysicien. Par ce seul fait il aborde ces questions difficiles avec un esprit neuf, pour ainsi dire, sans être gêné par les brumes de la terminologie et de la scolastique dont les spécialistes arrivent si difficilement à se débarrasser. Moins chargés de passé, les problèmes lui apparaissent plus simples et surtout il les aborde avec plus d'audace, et aussi, peut-être, plus de clarté. ${ }^{16}$

De même, en 1922, quand il présente la théorie de la dérive des continents au public français, le géologue suisse Élie Gagnebin souligne que Wegener «n'est point un géologue; il est géophysicien», omettant de préciser qu'il est géophysicien des enveloppes externes du globe. Il ajoute plus loin:

J'insiste encore sur le fait que Wegener n'est qu'un géologue d'occasion; il faut accepter sa gaucherie et se tenir de pousser les hauts cris lorsqu'elle se manifeste. Les absurdités apparentes de sa théorie, il n'est pas certain qu'elles condamnent l'idée fondamentale ni même qu'elles aient grande importance. ${ }^{17}$

De même que leurs collègues anglo-saxons,$^{18}$ aucun des commentateurs français de Wegener ne le considère réellement comme un membre de sa discipline. Le géologue Jacques Bourcart l'identifie en tant que météorologiste, les géologues Pierre Termier, Maurice Gignoux ou Charles Jacob le voient en géophysicien, tout comme le paléontologue Léonce Joleaud ou le zoologue Louis Fage. Cas extrême, le géophysicien Edmond Rothé, sismologue, le voit quant à lui ... en géologue. Soulignant que le savant allemand n'est pas géologue mais géophysicien, le géologue suisse John Leuba, explique, tirant parti de connaissances acquises en psychanalyse, que si la théorie de la dérive n'a pas conquis l'adhésion, «cela tient sans doute à ce fait psychologique si banal, que l'on est porté à n'accepter que les seules idées émises par les spécialistes». ${ }^{19}$ Wegener est donc toujours renvoyé à une autre discipline; jamais il n'est considéré comme spécialiste de ce qu'il expose. En cela, nous semble-t-il, il participe d'une science d'amateur.

\section{Une théorie trop commode?}

Les simplifications qu'il opère font l'objet de jugements contradictoires. Alors que Gignoux les valorise, Pierre Termier, membre de l'Institut, juge la théorie «trop commode»:

16 Gignoux 1925, 140.

17 Gagnebin 1922, 293.

18 Marvin 1973.

19 Leuba 1925, 208. 
Il est de l'essence même d'une pareille théorie d'être extrêmement commode: avantage qui ne va pas sans un grave danger, le danger de faire croire aux esprits superficiels que les énigmes sont résolues alors qu'elles sont simplement déplacées et remplacées par une énigme plus générale beaucoup plus impénétrable [...].

La théorie de Wegener est pour moi un beau rêve, un rêve de grand poète. On cherche à l'étreindre et l'on s'aperçoit que l'on n'a dans les bras qu'un peu de vapeur ou de fumée; elle est à la fois séduisante et insaisissable. ${ }^{20}$

La théorie elle-même est diversement appréciée. Lors d'un débat organisé à la Société géologique de France, en 1923, les positions des spécialistes divergent. ${ }^{21}$ Pour le zoologue du Muséum national d'histoire naturelle, Louis Germain, «la théorie de A. Wegener est en contradiction absolue avec les faits observés». Il estime que «dans l'état de nos connaissances, la distribution géographique des animaux se comprend seulement si on admet l'existence de ponts continentaux aujourd'hui disparus sous les mers». Pour son collègue, le zoologue Louis Fage, la prudence s'impose lorsqu'il s'agit de s'appuyer sur la distribution géographique des animaux, car ne peuvent être mobilisés que les groupes dont la systématique est parfaitement établie. Or, selon lui, «tel ne semble pas être le cas en particulier de ceux choisis comme exemples par les partisans de la dérive des continents». Dans l'exposé qui introduit le débat, Léonce Joleaud est cependant moins catégorique: il estime que la théorie de Wegener rend compte de certaines anomalies de la paléoclimatologie et qu'«elle peut contribuer à élucider certaines énigmes de la tectonique». Dans son domaine de compétence, la paléontologie, Joleaud pense que la théorie de Wegener peut aider à résoudre certains problèmes de la répartition des flores et des faunes fossiles, sans rendre compte, cependant, de toute la complexité des phénomènes de biogéographie. Joleaud propose, en conséquence, d'amender la théorie wegenérienne en imaginant un mouvement en accordéon des continents, «une sorte de mouvement oscillatoire de Madagascar se détachant de l'Inde pour aller se coller à l'Afrique, puis s'éloignant, se rapprochant ensuite, et s'écartant à nouveau du continent noir».22

En 1924, le tectonicien suisse de Neuchâtel, Émile Argand (1879-1940), va plus loin. Il publie sa Tectonique de l'Asie, un long texte de deux cents pages qui prolonge une conférence donnée lors du Congrès géologique de Bruxelles deux ans plus tôt. ${ }^{23}$ Après avoir exposé les données de terrain disponibles sur l'Asie, il explique que depuis 1915 et surtout 1918, il a longuement scruté le degré de crédibilité des hypothèses de Wegener. Contraire-

20 Termier 1924, 260 et 266.

21 Joleaud 1923.

22 Joleaud 1923, 238.

23 Argand 1924. 
ment à Termier qui érigeait en défaut la trop grande «commodité» de la théorie des translations continentales, Argand en fait une qualité. Il souligne son «extrême flexibilité jointe à une grand richesse en tours opératoires» et explique: «On pense tenir une objection décisive, encore un coup et tout va craquer, mais rien ne craque: on n'a oublié qu'un ou plusieurs tours. C'est la résistance protéenne d'un univers plastique.»²4 Dans les années 1920-1930, alors que la plupart des géologues centrent leurs analyses sur les mouvements verticaux de l'écorce terrestre, certains, tel Argand, estiment donc que les propositions de Wegener peuvent ouvrir la voie à de nouveaux programmes de recherche, au sens que Lakatos donne à cette expression..$^{25}$

Même s'ils estiment que Wegener n'est pas spécialiste, Maurice Gignoux, Charles Jacob ou Jacques Bourcart soulignent que la nouvelle théorie permet de relier de manière simple diverses données de terrain que les théories antérieures peinent à expliquer, en proposant des hypothèses qu'il convient d'éprouver. ${ }^{26}$ D'autres envisagent que leur discipline permettra de trancher le débat. En 1926, Paul-Louis Mercanton, glaciologue et physicien suisse, propose d'utiliser des mesures paléomagnétiques pour vérifier les déplacements des continents. ${ }^{27}$ Georges Perrier, secrétaire général de l'Union géodésique et géophysique internationale, estime que, bientôt, ses collègues mettront à la disposition des géologues d'abondants matériaux d'une haute exactitude sur la fixité ou la variabilité des longitudes. ${ }^{28} \mathrm{La}$ détermination et la révision des longitudes occupent aussi les astronomes du Bureau international de l'heure, au sein de l'Union astronomique internationale.$^{29}$ Le Congrès astronomique international de Boston, en 1932, émet le vœu que «des mesures spéciales des différences de longitude [soient] exécutées entre des stations où cette dérive des continents pourrait le mieux être constatée, c'est-à-dire entre l'Écosse et le Groenland, Madagascar et la Côte de Mozambique, l'Inde et les îles de la Sonde». ${ }^{30}$ Bien que Wegener ne soit pas considéré comme un spécialiste, ces jugements intègrent sa théorie dans «la science en marche».

24 Argand 1924, 292.

25 Lakatos 1994.

26 Gignoux 1925; Jacob 1925; Bourcart 1926.

27 Mercanton 1926.

28 Perrier 1923, 267.

29 Voir, par exemple, Stoyko 1932.

30 Houllevigue 1935, 351-352. 


\section{Des amateurs dans le sillage de Wegener}

Les débats sur les hypothèses de Wegener permettent à d'autres acteurs de la science, moins établis du point de vue institutionnel, de formuler leurs propres théories.

En 1924, dans un article de la Revue scientifique, ${ }^{31}$ Jacques Bourcart met l'accent sur un «précurseur» de Wegener, suscitant l'intérêt pour un personnage resté jusqu'alors dans l'ombre, un véritable acteur de la science des amateurs. Roberto Mantovani (1854-1933) est un violoniste, professeur de musique, ${ }^{32}$ qui a publié deux articles, l'un en 1889 dans le Bulletin de la Société des arts et sciences de La Réunion, ${ }^{33}$ puis l'autre en 1909 dans la revue de vulgarisation Je m'instruis. La science pour tous, ${ }^{34}$ sur l'idée d'une dilatation planétaire pour expliquer la disposition des continents et des chaînes de montagnes. Bourcart, méconnaissant l'article de 1889, indique que Mantovani avait formulé en 1909 l'hypothèse d'une séparation des continents de l'hémisphère sud par un mécanisme original, l'expansion du globe terrestre. Quelques mois après la publication de l'article de Bourcart, les portes de la Société géologique de France s'ouvrent à Mantovani qui y fait deux communications, en 1924 sur «Les points de contact entre la théorie de la dilatation planétaire et la théorie de la dérive des continents de Wegener» et en 1927 sur «L'Atlantide et la découverte de la dilatation planétaire». ${ }^{35} \mathrm{Il}$ aura quelques échanges épistolaires avec Wegener qui inclura l'article de 1909 dans la bibliographie de la dernière édition de son ouvrage en 1929.36

Dans le même ordre d'idée, Émile Belot (1857-1944), un ingénieur qui a élaboré une théorie personnelle sur la formation des planètes, profite du succès médiatique de la théorie des translations pour développer, en opposition à Wegener, sa propre hypothèse d'une cosmogonie «dualiste» et «tourbillonnaire» déjà exposée depuis une vingtaine d'années. ${ }^{37}$ Polytechnicien, théoricien de l'organisation industrielle et de l'automatisation du travail, il intervient dans plusieurs revues comme la Revue scientifique, ${ }^{38}$ La Science et

31 Bourcart 1924.

32 Arrivé en 1874 avec une troupe de théâtre, Mantovani s'est installé sur l'île de La Réunion où il fut aussi consul d'Italie et correspondant de diverses sociétés savantes italiennes, avant de s'installer en Bretagne au tournant du siècle. Il y poursuivit à la fois ses activités de musicien et ses conférences scientifiques sur les tremblements de terre et les volcans. Voir Gohau 1990 et Scalera 2009.

33 Mantovani 1889.

34 Mantovani 1909.

35 Mantovani 1924; Mantovani 1927.

36 Scalera 2009.

37 Fages 2012, 281-296.

38 Belot 1924a. 
la Vie ${ }^{39}$ la Revue générale des sciences pures et appliquées ${ }^{40}$ et dans quelques institutions comme l'Académie des sciences où ses notes de géologie sont présentées par Pierre Termier. Dans sa communication du 4 février 1924, il signale les insuffisances explicatives «des théories assez hardies» de l'auteur allemand, lequel «ne fait [pas] mention de l'eau comme agent d'action dans l'architecture continentale». Il estime avoir résolu dès 1914 les problèmes posés par Wegener et élucidé les questions laissées sans réponse. Comme Wegener, il en vient à admettre que certains socles continentaux ont pu se déplacer, mais dans des conditions différentes de celles qu'imagine l'Allemand: «Aucun socle ne peut dériver autrement qu'entraîné par le sima en mouvement dans lequel il est profondément ancré», souligne-t-il. ${ }^{41}$

D'autres auteurs encore, tels l'ingénieur Pierre Dive (1899-1960) ${ }^{42}$ ou le médecin militaire Philibert Russo (1885-1965), ${ }^{43}$ utilisent la théorie de Wegener pour construire leur propre vision de la constitution du globe terrestre et des processus qui l'affectent. Ainsi, les débats autour de la théorie de Wegener ont-ils permis à des amateurs d'accéder aux tribunes de plusieurs institutions et de diffuser leurs conceptions. Leurs contributions ont alimenté les discussions des années 1920 et jusqu'aux années 1940, dans un contexte où une très large majorité se dessine parmi les géologues pour rejeter, faute de preuves, l'hypothèse de la dérive des continents.

\section{Comme des glaçons à la surface de l'eau: une théorie aisément vulgarisable}

Certaines caractéristiques de la théorie de Wegener favorisent sa diffusion au-delà des cercles scientifiques: dans les années 1920, la dérive des continents devient un sujet «à la mode».

Présentée comme une grille d'explication globale de phénomènes naturels divers, comme le volcanisme et les séismes, la théorie trouve un écho dans des ouvrages grand public écrits par des géologues, tels Leuba en $1925 \mathrm{chez}$ Armand Colin ${ }^{44}$ ou Bourcart en 1926, aux éditions Quillet. Présentée sous un jour favorable, cette théorie est mobilisée, en particulier, pour expliquer la formation des montagnes et la localisation des régions volcaniques: «Bien que la part de l'hypothèse soit considérable dans cette grande théorie, elle est

39 Belot 1924b.

40 Belot 1924c.

41 Belot 1924d.

42 Dive 1933; Dive 1934.

43 Russo 1930; Russo 1933.

44 Leuba 1938, 154-155. 
appelée à donner un nouvel enchaînement de tous les faits connus et ainsi à susciter des travaux pour éprouver sa valeur.» ${ }^{45}$ Par ailleurs, les livres à succès de Pierre Termier contribuent aussi à diffuser le débat sur la théorie dans un large public. ${ }^{46}$

Les chroniques scientifiques des journaux et les revues scientifiques destinées au grand public - notamment aux plus jeunes - ne sont pas en reste. Elles diffusent l'image de continents s'en allant à la surface du globe, comme flottent des glaçons à la surface de l'eau. Dans la rubrique qu'il tient dans l'hebdomadaire illustré Ric et Rac, le biologiste et vulgarisateur Georges Colomb signe, le 24 février 1934, un texte intitulé «Les continents en balade», ${ }^{47}$ puis, en 1937, il présente à nouveau Wegener et sa théorie dans un article sur la vie et la reproduction des anguilles..$^{48}$ Lauteur fait partager à son lectorat, son émerveillement devant la fécondité explicative qui émerge de l'association de deux données scientifiques que rien, a priori, ne rapproche: la reproduction des anguilles européennes dans la lointaine mer des Sargasses d'une part, et la dérive des continents, d'autre part. Les anguilles auraient conservé la mémoire d'un temps où leur lieu de ponte restait encore proche des côtes européennes à une époque où l'Atlantique n'existait pas. Or nous savons que la rencontre de deux domaines différents renforce la validité de la théorie, au moins dans l'esprit des lecteurs. ${ }^{49}$

La théorie de Wegener apparaît également dans la littérature populaire, comme en témoigne un épisode de la célèbre série Le Saint, de l'écrivain anglais Leslie Charteris, qui met en scène le personnage de Simon Templar. Dans La Justice du Saint, épisode publié en Grande-Bretagne en 1936, des pirates menés par Kurt Vogel comptent détourner l'invention du professeur Yule, le bathystol, un scaphandrier conçu pour «vérifier l'exactitude de la théorie de Wegener sur le glissement des masses continentales», ${ }^{50}$ afin de devancer la récupération, par les assureurs, d'un trésor coulé. En France, ce roman est paru en feuilleton dans l'hebdomadaire Ric et Rac en 1938. Dans un tout autre genre, le poète Pierre Guéguen (1889-1965) compose un poème scientifique intitulé SI-AL, SI-MA, NI-FE en expliquant:

SI-AL, c'est notre mère la Terre, ma périphérie: peau de SI-lice et d'AL-umine; SI-lice et MA-gnésie, sous-tend SI-AL, comme une cave la maison. Le vin de lave y crève la futaille des volcans; NI-FE, NI-ckel et FE-r, fruit caché sous l'écorche, c'est la pulpe de feu, rigide à la pression de fabuleuses atmosphères. ${ }^{51}$

45 Bourcart 1926, 37.

46 Termier 1925; Termier 1929.

47 Colomb 1934.

48 Colomb 1937.

49 Voir Laudan R. et Laudan L. 1989.

50 Charteris 1938.

51 Guéguen 1929. 
Et si la thèse de Wegener diffuse ainsi, c'est qu'elle est relativement facile à appréhender, notamment parce qu'elle peut être aisément résumée par l'analogie des glaçons qui flottent sur l'eau. Tel n'est pas le cas d'autres théories, telle celle des géosynclinaux à laquelle se réfèrent alors les géologues. Les schémas qui permettent de l'illustrer sont complexes: sur un fond de carte
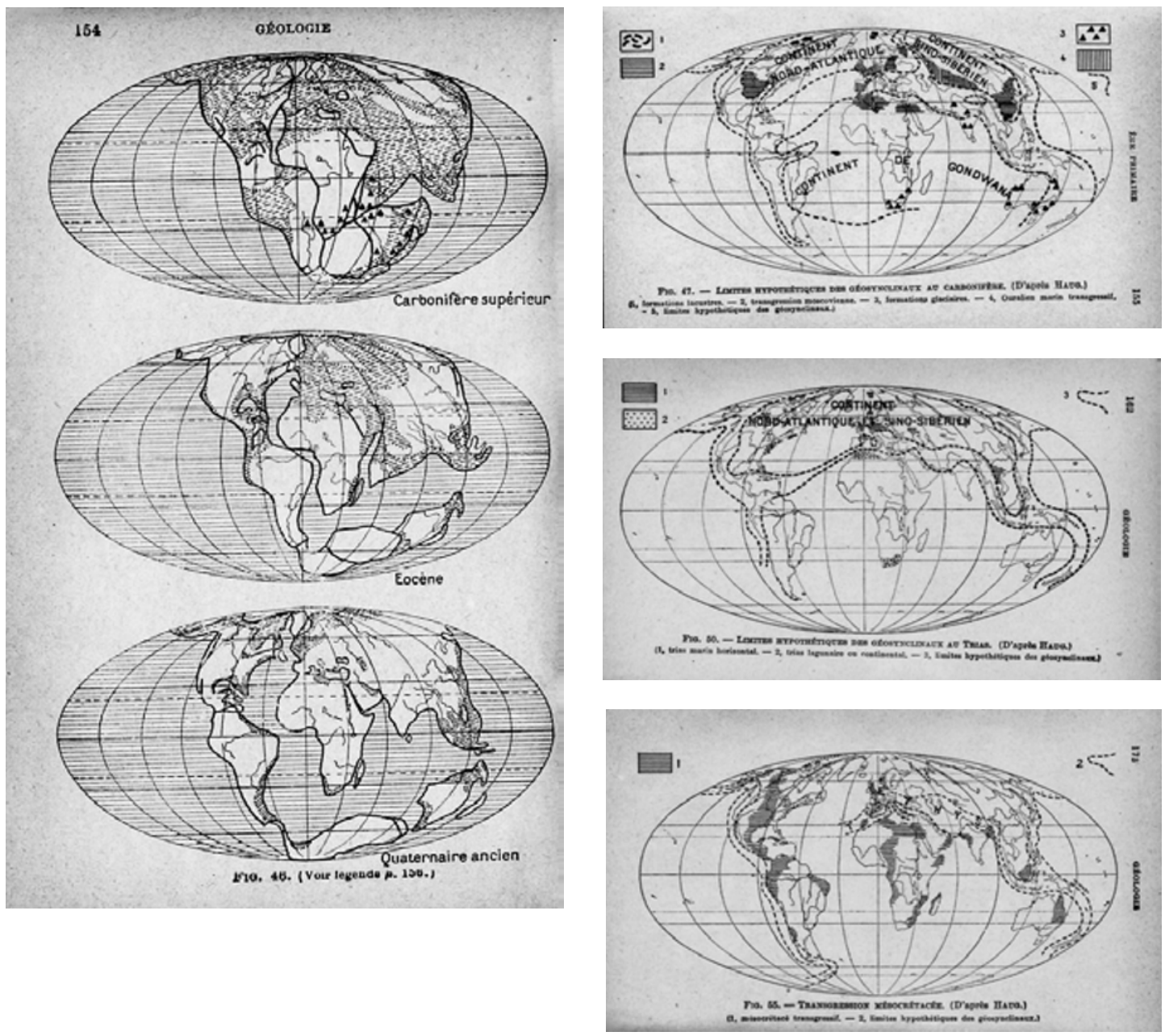

Leuba, John, 1938. Introduction à la géologie. Librairie Armand Colin, Collection Armand Colin (section de géographie),

Paris, $5^{\mathrm{e}}$ édition (1 $\left.{ }^{\mathrm{e}} \mathrm{Ed} .1925\right), 216 \mathrm{p}$. Extraits pages 154, 155, 162, 172, 181.

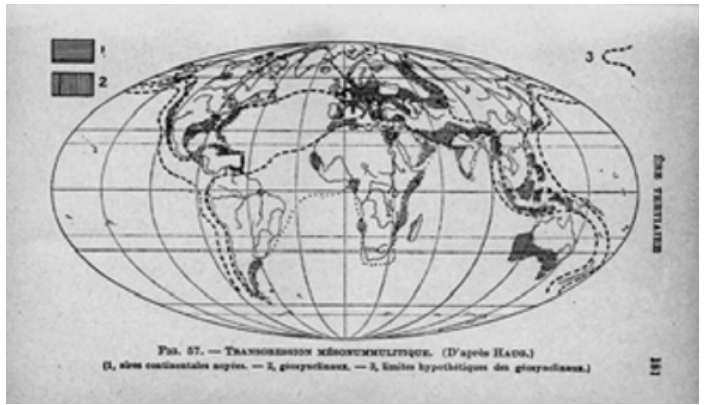

Morcellement du bloc continental, la Pangée du Carbonifère, suivi d'une derive des continents selon Wegener (à gauche); limites successives des continents et des geosyndinaux au cours des temps géologiques selon Haug (à droite). 
de la répartition actuelle des masses continentales, des traits superposés matérialisent les contours, changeants au cours du temps, des grands domaines continentaux. Au contraire, les cartes dessinées par Wegener et largement reprises par les vulgarisateurs, font découvrir une évolution simple et logique de l'écorce terrestre conduisant à la disposition actuelle des continents: on reconnait, dès le Carbonifère, la forme des continents qui s'emboitent alors en un continent unique puis se séparent, en conservant la forme qu'on leur connait aujourd'hui, pour occuper leurs emplacements actuels. La dérive est facilement visualisable.

\section{Science scolaire / science universitaire}

Sans doute, ces représentations sont-elles très pédagogiques puisque la théorie wegenérienne connaît une étonnante persistance dans les manuels scolaires. Elle y trouve une légitimité, alors même qu'elle est délaissée par les géologues universitaires.

Le géographe René Ozouf expose la dérive des continents dans Le Journal des Instituteurs et des Institutrices, en 1928. Il se projette aussi dans l'avenir:

Les progrès de la radiotélégraphie, en donnant à la mesure des longitudes une précision, à un petit nombre de mètres près, permettront-ils, en dépit de la prodigieuse lenteur avec laquelle se feraient les mouvements de dérive des continents, de nous rendre compte expérimentalement de l'existence de ces mouvements? C'est le secret de demain. ${ }^{52}$

L'intérêt du monde de l'enseignement ne se dément pas tout au long des années 1930 et même au-delà. En 1931, Élie Lazerges, inspecteur général de l'Instruction publique, présente dans Les grands problèmes de la Biologie et de la Géologie «l'hypothèse ingénieuse et séduisante» que propose Wegener «pour expliquer d'une manière simple en même temps que les transformations de la géographie au cours des temps géologiques, la formation des montagnes, et pour relier à une même cause tout un ensemble de phénomènes géologiques qu'on a l'habitude de considérer comme distincts et dont la cause reste obscure» ${ }^{53} \mathrm{En}$ 1932, c'est un professeur de mathématique dans un lycée malgache qui, à la faveur d'un discours de remise de prix, met à l'honneur la dérive appliquée à Madagascar. ${ }^{54}$ Plus tard, le rapporteur de l'épreuve écrite de géographie du Certificat d'aptitude au professorat des Écoles normales et des Écoles primaires supérieures, souligne, pour la

52 Ozouf $1928,140$.

53 Lazerges 1931, 125-126.

54 Vinot 1932. 
session de 1934, que certains candidats, «ont à cœur de montrer qu'ils connaissent la géographie générale»:

Aussi l'étale-t-on partout; on la porte en quelque sorte en écharpe et il n'est pas rare de trouver exposée[s], (...) à propos d'un massif charrié, les hypothèses de Wegener. Il y a là un pédantisme ridicule. C'est d'autant plus fâcheux que la plupart de ceux qui font ainsi étalage de connaissances mêlent à leur science de grossières erreurs..$^{55}$

En 1943, le géographe André Allix, qui fut correcteur de l'agrégation, se souvient qu'avant la guerre certains candidats «malax[ai]ent gauchement l'isostasie, la dérive des continents, les idées d'Émile Argand», ${ }^{56}$ sans mâ̂triser les bases de la discipline.

Dans les années 1940, les auteurs de manuels présentent encore, pour certains d'entre eux, la théorie de Wegener comme une théorie récente, capable d'expliquer des phénomènes de diverse nature. Dans son édition de 1946, le manuel de $4^{\text {e }}$ Géologie Botanique de Marcel Oria, professeur de sciences naturelles au Lycée de Versailles, introduit un schéma «montrant comment la théorie de la dérive des continents rend compte de la formation des chaînes de montagnes (côtières et géosynclinales)». ${ }^{57}$ En 1947, le manuel de 4 e Sciences Naturelles d'Albert Obré décrit la théorie comme acquise, sans le moindre conditionnel (soulignant, par l'italique, les noms de l'auteur et de sa théorie que l'élève devra retenir):

Les continents peuvent être assimilés à des radeaux de SIAL (lithosphère) qui flottent, comme des iceberg, en s'enfonçant plus ou moins dans le SIMA visqueux; ils se déplacent lentement vers l'Ouest avec des vitesses variables; c'est ce que Wegener appelle la dérive des continents. ${ }^{58}$

En 1953 encore, Pierre Vincent, dans son manuel pour les classes de $4^{\mathrm{e}}$, affirme que «la formation des montagnes s'explique assez bien par la théorie de la dérive des continents» et se contente de signaler, sans commenter, que «des nombreuses mesures faites ces dernières années, aucune n'a apporté la preuve de l'existence d'un tel mouvement; aussi beaucoup de géologues refusent-ils d'admettre la théorie de Wegener». ${ }^{59}$ En 1954, l'idée de la dérive persiste dans le manuel de Jean-Jacques Humbert, professeur de sciences naturelles au Lycée de Sceaux. Les critiques concernant l'absence de forces suffisantes pour mettre en mouvement les masses continentales sont évoquées. À la question «d'autres forces, encore inconnues, seraient-elles encore à l'œuvre?», il est répondu que «quelques savants pensent qu'il faudrait faire

55 Anonyme 1935, 282.

56 Allix 1943, 217.

57 Oria 1946, 228.

58 Obré 1947, 262.

59 Vincent 1953, 236. 
intervenir des courants dans le magma; très lents, mais très puissants, [qui] entraîneraient irrésistiblement les radeaux continentaux».60 L'auteur fait allusion à des hypothèses qui avaient été avancées dès le milieu des années 1920 sans rencontrer de réelle adhésion chez les géologues.

Ce survol de l'édition scolaire jusqu'au milieu du siècle montre que les auteurs de manuels véhiculent dans le jeune public une vision du globe terrestre que ne partagent plus les professionnels du sujet, les géologues universitaires. On peut penser qu'il s'agit de réminiscences des cours qu'ils avaient reçus eux-mêmes. Les programmes de 1947,61 qui renvoient aux instructions de 1938, précisent pourtant à propos des «phénomènes géologiques actuels»: «Il s'agit non d'énumérer, mais de faire comprendre (quand il est connu) le mécanisme des phénomènes. On devra aussi bien éviter les hypothèses encore discutées, que les conclusions périmées ou simplistes.» ${ }^{62}$ Les auteurs ont donc passé outre la consigne officielle en exposant une théorie considérée par les géologues au mieux comme simpliste, au pire comme périmée. Cette théorie présente en effet l'avantage d'être tout à la fois englobante et compréhensible de manière presque intuitive, par les enseignants et par les jeunes élèves de la classe de quatrième, grâce à la métaphore de blocs continentaux flottant sur une couche plus dense mais plus fluide, à la façon des glaçons dans un verre d'eau. Comme l'écrit Lazerges:

Quel que soit l'avenir que les résultats de la géophysique et de la géologie réservent à la théorie de Wegener, elle n'en apparaît pas moins comme une hypothèse à la fois grandiose et séduisante parce qu'elle donne de phénomènes mystérieux et, tout au moins en apparence, complexes, une explication à la fois générale et simple. ${ }^{63}$

Les auteurs de manuels apparaissent certes comme des spécialistes aux yeux des enseignants, comme ces derniers le paraissent aux yeux de leurs élèves. Dans la réalité, ces professionnels de l'enseignement peuvent être considérés comme des amateurs face aux géologues universitaires: pour eux, comme pour les vulgarisateurs, la science de Wegener, contrairement à celle des spécialistes universitaires, présente l'avantage de pouvoir être aisément traduite en termes simples et en images qui frappent les imaginations. Son pouvoir explicatif englobant lui confère la capacité de susciter l'admiration, voire l'émerveillement.

60 Humbert 1954, 212.

61 Bulletin officiel du ministère de l'Éducation nationale, 1947, 504.

62 Ministère de l'Éducation nationale 1938, 206.

63 Lazerges 1931, 169. 


\section{Science d'amateur et (para-)science}

Pour des raisons similaires, la théorie de la dérive des continents a pu être mise au service d'hypothèses que l'on peut qualifier de para-scientifiques.

En parallèle à un renouveau littéraire, lié notamment au succès de L'Atlantide de Pierre Benoit en 1919, l'époque voit la résurgence du mythe atlantéen et la naissance d'une «Atlantologie» ${ }^{64}$ qui se veut «science» par la mobilisation de connaissances naturalistes sur les géographies passées et sur l'évolution de la croûte terrestre d'une part, et, d'autre part, la mobilisation de connaissances anthropologiques et préhistoriennes sur le passé, sur les caractéristiques et sur les légendes des groupes humains, d'Afrique du Nord en particulier. Plusieurs courants débattent à propos de l'Atlantide, privilégiant approche «positive» des données d'observation ou quête spiritualiste.

Un certain nombre de naturalistes se sont penchés sur l'existence et sur la localisation de l'île décrite par Platon. C'est le cas de Termier, Germain ou Joleaud, pour n'en citer que quelques-uns. ${ }^{65}$ D'autres universitaires, des géographes, botanistes, historiens, anthropologues ou littéraires se sont intéressés au sujet. Mais celui-ci a attiré aussi une nébuleuse d'auteurs qui n'ont pas toujours accès aux tribunes et publications scientifiques. Réunis au sein d'associations plus ou moins imprégnées d'ésotérisme, ils sont attentifs à la nouvelle conception de l'histoire des continents proposée par Wegener. Car, alors que la thèse des effondrements et des ponts continentaux entérinait l'idée du naufrage d'un continent dans les eaux de l'océan et donc l'impossibilité d'en retrouver les traces, la disparition d'une terre sous les flots n'est pas compatible avec la conception de continents uniquement animés de mouvements de translation. La disparition de l'Atlantide ne serait donc plus une submersion mais un éloignement de sa position initiale, par dérive, avec les habitants qui la peuplent. Il devient possible de partir à sa recherche.

En décembre 1923, dans une chronique de la revue La Géographie, Jean Gattefossé présente deux ouvrages récents sur l'Atlantide, ${ }^{66}$ l'un de Michel Manzi et l'autre de son propre frère, René-Maurice Gattefossé, qui, sur la base d'une interprétation de données anthropologiques, envisage une Atlantide atlantique formant un pont entre l'Afrique, l'Europe et l'Amérique. Il dresse le plan des futures recherches sur le problème et propose «de l'examiner aux lumières apportées par Gattefossé et par Wegener». En plus

64 Une «Société d'études atlantéennes» est créée en 1926, qui donne naissance, après scission en 1927, à un groupement «Les amis d'Atlantis» et une revue Atlantis. Sur le contexte de la naissance de l'atlantologie et les débats de cette époque, voir Lagrange 2008.

65 Termier 1913; Germain 1913; Germain 1924a; Germain 1924b; Joleaud 1928; Joleaud 1929. 66 Manzi 1922, Gattefossé R.-M. 1923. 
d'y trouver matière à réflexion, les lecteurs «ne s'étonneront pas de constater que la théorie de la dérive des continents, loin de supprimer l'hypothèse de l'Atlantide, la confirme et la renforce au contraire». ${ }^{67}$ La théorie de Wegener est donc «récupérée» dans un cadre bien éloigné du champ scientifique, associant le savant allemand à un auteur dont l'œuvre, selon J. Gattefossé, «donne implicitement raison aux travaux des occultistes et confirme les traditions ésotériques». ${ }^{68}$ Quelques années plus tard, Gattefossé écrit que «le problème de l'Atlantide est étroitement lié aux théories de Wegener et d'Émile Belot; [...] je reste personnellement persuadé que c'est de leur future conjonction harmonieuse que surgiront de nouvelles hypothèses de travail, fructueuses en résultats positifs pour les atlantologues notamment». ${ }^{6}$ Plusieurs autres amateurs d'Atlantide discuteront la thèse de Wegener, la mobilisant avec plus ou moins d'enthousiasme. ${ }^{70}$

Dans sa chronique de 1923, J. Gattefossé, en bon patriote, identifie un précurseur français à Wegener:

Que l'on nous permette en passant de revendiquer la théorie de la dérive des continents, comme une idée essentiellement française, dans son ensemble, puisqu'elle fut publiée pour la première fois, avec des cartes explicites par Snider-Pellegrini dans son ouvrage si curieux: La création et les mystères dévoilés, ouvrage où l'on expose clairement la nature de tous les êtres, l'origine de l'Amérique, etc. ${ }^{71}$

Antonio Snider-Pellegrini (1802-1885), un italo-américain vivant à Paris, est généralement présenté comme un scientifique ou un géographe. ${ }^{72}$ En 1859, dans La création et ses mystères dévoilés, ${ }^{73}$ il explique la disposition actuelle des continents par les effets d'une séparation cataclysmique de masses continentales jusqu'alors rassemblées en une seule. Son texte est accompagné de deux gravures du globe terrestre présentant la position des continents avant et après la rupture. Cet amateur, qui organise son ouvrage autour des six jours de la Création qu'il assimile à des époques et dans lequel il tente de concilier les connaissances sur le monde avec les écrits bibliques, se trouve, en quelque sorte, élevé à la dignité scientifique en tant que précurseur de Wegener. Les atlantologues, pour leur part, préfèrent le premier au second. Ses thèses correspondent mieux à leurs propres préoccupations teintées d'un certain ésotérisme.

67 Gattefossé J. 1923, 597.

68 Gattefossé J. 1923, 597.

69 Gattefossé 1927, 732.

70 Voir Delvaux 1926; Callet 1927; Cottevieille-Giraudet 1930; Barbarin 1938.

71 Gattefossé J. 1923, 598.

72 Robert M. Wood indique toutefois que Snider-Pellegrini (1802-1885) n'est présenté comme scientifique que parce qu'il est cité, parmi d'autres scientifiques en 1861 dans l'ouvrage de John Henry Pepper (1821-1900), The playbook of metals. Wood 1985, 38.

73 Snider 1859, 309. 
C'est dans le contexte d'un courant qui pourrait être qualifié de (para-) science $^{74}$ que Snider-Pellegrini est associé à la théorie wegenérienne. Or, quittant la sphère des amateurs d'Atlantide, le nom de Snider-Pellegrini gagne le monde des professionnels de la géologie lorsqu'il est cité comme précurseur des idées de la dérive en 1944 par le géologue britannique Arthur Holmes. Son traité, Principles of Physical Geology, reproduit les deux gravures du globe. ${ }^{75}$ Snider-Pellegrini sera confirmé ultérieurement par des historiens de la géologie ${ }^{76}$ comme précurseur de Wegener. Albert Carozzi écrit, par exemple, en 1969:

Snider apparaît comme le premier précurseur de l'idée des dérives continentales, ayant assemblé et disloqué les continents à peu près de la même façon que Taylor (1908) et Wegener (1910) le firent au début du vingtième siècle. ${ }^{77}$

Wegener toutefois semble avoir ignoré l'existence des publications de SniderPellegrini. Aucune référence à cet auteur n'apparaît dans l'édition de 1922 traduite. Le nom reste absent, malgré les mises à jour opérées par Wegener, dans la dernière édition de 1929.

La question de l'Atlantide a donc pu s'alimenter des débats scientifiques qui ont surgi avec la nouvelle conception géologique venue d'Allemagne. Ainsi le signale un correspondant de la revue La pensée française, en 1926:

Que vaut cette théorie nouvelle? L'avenir le dira; la télégraphie sans fil met aujourd'hui, à notre disposition un moyen puissant pour la détermination rigoureuse des longitudes. Quoi qu'il en soit, en ce moment, c'est «l'affaire Wegener» qui est à la barre du tribunal de la science, et le jugement qui sera rendu simplifiera peut-être beaucoup l'instruction de l'affaire Atlantide. ${ }^{78}$

Mais la théorie de la dérive des continents a pu être mobilisée dans d'autres cadres relevant de ce que la plupart des contemporains considéraient comme para-scientifique. Loin des milieux académiques, Fernand Crombette (18801970), un employé de l'administration des Postes, ${ }^{79}$ inspiré par une lecture littérale du texte biblique, ${ }^{80}$ estime nécessaire de placer Jérusalem au centre des terres émergées. Il appuie cette recomposition géographique sur Wegener, tout en s'en éloignant sur certains points:

Les aperçus que la reconstitution morphologique a pu nous offrir sur la géologie et la tectonique, dont la plupart constituent de véritables révélations, sont loin d'être les seuls que puisse offrir la restauration de la Terre telle que nous l'avons réalisée. Il va sans dire que les

74 Pour une discussion de ces aspects, voir Lagrange 2008.

75 Holmes 1944, 489-490.

76 Carozzi 1969; Marvin 1973; Wood 1985; Le Grand 1988; Oreskes 1999; Lewis 2002.

77 Carozzi 1969, 178.

78 Butavand 1926.

79 Derose 1994.

80 «Cependant, Dieu, notre Roi, dès avant les siècles, a opéré le salut au milieu de la terre», verset 12 du Psaume 73 de la Vulgate. 
réalisations paléontologiques dont se parait la théorie de Wegener ont encore plus de force dans notre reconstitution, bien plus exacte que la sienne. ${ }^{81}$

Contestant la dérive lente des continents envisagée par le savant allemand, Crombette imagine un transport brutal, «sur à peu près 90 jours, comme l'indique la Bible: non par suite de phénomènes rentrant dans le domaine des causes actuelles, mais par suite d'une immense catastrophe», ${ }^{82}$ le déluge universel. Ainsi, Crombette fait-il entrer la nouvelle hypothèse sur le globe dans le cadre littéral du texte biblique. Le caractère «trop commode» de la théorie wegenérienne que dénonçait Termier, ou encore «protéen» qu'appréciait Argand, autorise l'amateur de géographie biblique à déplacer à sa guise les pièces du puzzle continental pour obtenir une figure de la Terre compatible avec son exégèse.

\section{Conclusion}

La théorie tectonique de Wegener se situe difficilement dans un classement où nous distinguerions des sciences de spécialistes et d'amateurs. Dans la mesure où elle est globale, on peut la comparer à d'autres systèmes de même ampleur: celui d'Élie de Beaumont (et son réseau pentagonal), celui de Suess, avec ses effondrements, tous deux appuyés sur des œuvres importantes. Les auteurs de ces constructions ont tous été tenus, par leurs homologues, pour des spécialistes des sciences de la Terre.

Venant de la météorologie, une discipline éloignée de la géophysique profonde et de la tectonique, Wegener s'en distingue. Sans doute cela explique-t-il qu'il se soit heurté aux spécialistes des diverses branches de la géologie, incluant la paléontologie et la géophysique. Il n'a pu leur opposer une œuvre comparable à la Face de la Terre de Suess, ni même au Traité de géologie d'Émile Haug sur la théorie des géosynclinaux. Mais la théorie de la dérive elle-même, dans son ambition explicative et dans les données qu'elle mobilisait, ne se distingue pas par nature des leurs.

Pour maints spécialistes contemporains toutefois, la théorie de Wegener devait être rangée parmi les élucubrations de «poète». Elle avait un caractère des fausses sciences: elle puisait à de trop nombreuses disciplines que l'auteur ne maîtrisait pas et elle compensait l'approximation de ses preuves en les multipliant, comme si l'accumulation de médiocres preuves valait preuve solide. La transdisciplinarité et l'accumulation de données pouvaient être des défauts pour certains spécialistes, qui y voyaient sans doute plutôt une 
«non-disciplinarité» et un éclectisme suspects. Mais ils pouvaient aussi être une qualité pour de nombreux amateurs et vulgarisateurs qui pouvaient aisément comprendre les propositions théoriques de Wegener et s'émerveiller de leurs potentialités explicatives. C'est pourquoi sans doute, après la mort de son auteur et jusqu'au milieu du siècle, sa théorie se cantonna, comme on a vu, essentiellement aux domaines de la science des amateurs, des éducateurs et des vulgarisateurs.

\section{Bibliographie}

Anonyme, «Rapport sur les examens et concours», Bulletin de la Société des professeurs d'histoire et de géographie de l'enseignement public (1935) 25 (82): 271-294

Anonyme, «Modification des programmes des lycées et collèges. Arrêté du 18 avril 1947», Bulletin officiel du ministère de l'Éducation nationale (13) 504-515

Ministère de l'Éducation nationale, Instructions du 30 septembre 1938 relatives à l'application des arrêtés du 30 août 1937 et du 11 avril 1938 fixant les programmes de l'enseignement du second degré (Paris ${ }^{31956)}$

Allix, André, «La Géographie à l'agrégation, souvenirs d'un correcteur (suite et fin)», Les Études rhodaniennes 18 (3) (1943) 213-230

Argand, Émile, «La tectonique de l'Asie», Comptes rendus de la XIII esession Congrès géologique international Bruxelles 1922, fascicule 1 (Liège 1924) 171-372

Barbarin, Georges, La danse sur le volcan. Atlantide, Lémurie, continents futurs (Paris 1938)

Belot, Émile, «Le rôle de l'astro-géophysique en géologie», Revue scientifique 62 (27) (1924a) 752

Belot, Émile, «Genèse des continents et des mers et lois de l'architecture terrestre», La Science et la Vie 26 (89) (1924b) 423-426

Belot, Émile, «L'évolution et les résultats de la cosmogonie dualiste et tourbillonnaire», Revue générale des sciences pures et appliquées 35 (1924c) 463-467

Belot, Émile, «La genèse des continents et des mers. Note présentée par Pierre Termier», Comptes Rendus des Séances de l'Académie des Sciences 178 (1924d) $583-586$

Bertrand, Marcel, «Ilot triasique du Beausset (Var). Analogie avec le bassin houiller Franco-Belge et avec les Alpes de Glaris», Bulletin de la Société géologique de France $3^{e}$ série, 15 (1887) 667-702

Bourcart, Jacques, «Les origines de l'hypothèse de la dérive des continents», Revue scientifique 62 (18) (1924) 563-564

Bourcart, Jacques, La vie de la Terre illustrée (Paris 1926)

Butavand, F., «Encore la question de l'Atlantide», Les Annales politiques et littéraires 2264 (1926) 503

Callet, Charles, «À propos de l'Atlantide», Mercure de France 194 (690) (1927) 729_ 731

Carozzi Albert V., «À propos de la théorie des dérives continentales: Francis Bacon (1620), François Placet (1668), A. von Humboldt (1801) et A. Snider (1858)», 
Compte rendu des séances de la Société de physique et d'histoire naturelle de Genève 4 (3) (1969) 171-179

Charteris, Leslie, «La justice du Saint. Traduit de l'anglais par E. Michel-Tyl», Ric et Rac, Grand hebdomadaire pour tous 483 (1938) 10

Colomb, Georges, «Les continents en balade», Ric et Rac 258 (1934) 2

Colomb, Georges, «Le traditionalisme chez les bêtes», Ric et Rac 441 (1937) 2

Cottevieille-Giraudet, Rémy, «Les relations probables de l'Europe et de l'Amérique du Nord à l'âge du Renne», Congrès d'Anthropologie du Portugal (1930), dans: Poisson, G., L'Atlantide devant la science. Étude de préhistoire (Paris 1945) 238-245

Delvaux A., «Le problème de l'Atlantide et les Allemands», Mercure de France 185 (661) (1926) 258-262

Derose, Noël, «Synthèse de l'Essai de géographie [...] divine», Les Cahiers $d u$ CESHE 5 (1) (Tournai 1989)

Derose, Noël, Si le monde savait [...] Fernand Crombette, sa vie, son œuvre (Tournai 1994)

Dive, Pierre, La dérive des continents et les mouvements intra-telluriques (Paris 1933)

Dive, Pierre, «Conception nouvelle sur la structure interne du globe terrestre géomécanique et translation continentales», Annales de Géographie 43 (243) (1934) 225-235

Élie de Beaumont, Léonce, «Recherches sur quelques-unes des révolutions de la surface du globe», Annales de Sciences naturelles 18 (1828-1830) 5-25 et 284-416; $19,5-99$ et $177-240$

Fages, Volny, Les origines du monde. Cosmogonies scientifiques en France (18601920): acteurs, pratiques, représentations (Paris 2012)

Gagnebin, Élie, «La dérive des continents selon la théorie d'Alfred Wegener», Revue générale des sciences pures appliquées 33 (10) (1922) 293-304

Gattefossé, Jean, «À propos de l'Atlantide», La géographie 40 (5) (1923) 594-598

Gattefossé, Jean, «Notes et documents scientifiques. L'Atlantide», Mercure de France 195 (693) (1927) 731-734

Gattefossé, René-Maurice, La Vérité sur l'Atlantide (Lyon 1923)

Germain, Louis, «Les problèmes de l'Atlantide et la zoologie», Annales de Géographie 22 (123) (1913) 209-226

Germain, Louis, «L'Atlantide», Revue scientifique 62 (15) (1924d) 453-462

Germain, Louis, «L'Atlantide (suite)», Revue scientifique 62 (16) (1924b) 488-491

Gignoux, Maurice, «Les théories de Wegener. A propos de la traduction française de son ouvrage», Revue générale des sciences pures et appliquées 36 (5) (1925) $139-142$

Gohau, Gabriel, «Mantovani et sa théorie de la dilatation planétaire», Travaux $d u$ Comité français d'histoire de la géologie $3^{\mathrm{e}}$ série, 4 (6) (1990) 53-58

Guéguen, Pierre, «SI-AL, SI-MA, NI-FE», Les Maîtres de la Plume 5 (7) (1929) 24-25

Haug, Émile, «Les géosynclinaux et les aires continentales. Contribution à l'étude des transgressions et des régressions marines», Bulletin de la Société géologique de France $3^{\mathrm{e}}$ série, 28 (1900) 617-711

Haug, Émile, Traité de géologie. Volume 1: Les phénomènes géologiques (Paris 1907)

Holmes, Arthur, Principles of physical geology (London 1944)

Houllevigue, Louis, «Nos connaissances astronomiques dépendent de la puissance de l'outillage», La Science et la Vie 48 (221) (1935) 347-357 
Humbert, Jean-Jacques, Géologie, Classe de $4^{e}$ (Berlin, Paris ${ }^{11951, ~}{ }^{21954)}$

Jacob, Charles, «Les théories tectoniques nouvelles. E. Argand - A. Wegener», Annales de géographie 34 (188) (1925) 97-112.

Joleaud, Léonce, «Essai sur l'évolution des milieux géophysiques et biogéographiques (À propos de la théorie de Wegener sur l'origine des continents)», Bulletin de la Société géologique France 23 (4) (1923) 205-270

Joleaud, Léonce, L'Atlantide envisagée par le paléontologiste. Texte de la conférence faite à Paris le 30 avril (Paris 1928)

Joleaud, Léonce, «L'Atlantide. Esquisse de protohistoire eurafricaine», La Revue de Paris 36 (4) (1929) 886-898

Lagrange, Pierre, «Les controverses sur l'Atlantide (1925-1940) L'archéologie entre vraie et fausse science», dans: Voisenat, C. (éd.), Imaginaires archéologiques (Paris 2008) 233-277

Lakatos, Imre, Histoire et méthodologie des sciences. Programmes de recherche et reconstruction rationnelle (Paris 1994)

Laudan, Rachel/Larry Laudan, «Dominance and the disunity of method: solving the problems of innovation and consensus», Philosophy of science 56 (1989) 221-237

Lazerges, Élie, Les grands problèmes de Biologie et de la Géologie (Paris 1931)

Le Grand, Hommer E., Drifting Continents and Shifting Theories. The Modern Revolution in Geology and Scientific Change (Cambridge 1988)

Le Vigouroux, Philippe, «La Première Guerre mondiale et ses conséquences sur la réception de la théorie de Wegener en France», Travaux du Comité français d'histoire de la géologie 25 (2011) 187-206

Leuba, John, Introduction à la géologie (Paris ${ }^{11925,}{ }^{51938)}$

Lewis, Cherry L.E., «Arthur Holmes' unifying theory: from radioactivity to continental drift», dans: Oldroyd, D. R. (ed.), The Earth Inside and Out: Some Major Contributions to Geology in the Twentieth Century (London 2002) 167-183

Mantovani, Roberto, «Les fractures de l'écorce terrestre et la théorie de Laplace», Bulletin de la Société des arts et sciences de La Réunion (1889) 41-53

Mantovani, Roberto, «L'Antarctide», Je m'instruis. La science pour tous 38 (1909) 595-597

Mantovani, Roberto, «Les points de contact entre la théorie de la dilatation planétaire et la théorie de la dérive des continents de Wegener», Compte rendu sommaire des séances de la Société géologique de France 24 (16) (1924) 167-168

Mantovani, Roberto, «L'Atlantide et la découverte de la dilatation planétaire», Compte rendu sommaire des séances de la Société géologique de France 27 (4) (1927) 153-155

Manzi, Michel, Le livre de l'Atlantide (Paris 1922)

Marvin, Ursula B., Continental Drift. The Evolution of a Concept (City of Washington 1973)

McCoy, Roger M., Ending in Ice: the revolutionary idea and tragic expedition of Alfred Wegener (Oxford 2006)

Mercanton, Paul-Louis, «Inversion de l'inclinaison magnétique terrestre aux âges géologiques», Terrestrial Magnetism and Atmospheric Electricity 31 (4) (1926) 187-190 
Obré, Albert, Sciences naturelles Géologie, Classe de Quatrième (Paris 1947)

Oreskes Naomi, The Rejection of Continental Drift. Theory and Method in American Earth Science (New-York 1999)

Oria, Marcel, Géologie, Botanique, Classe de $4^{e}$ (Paris 1946)

Ozouf, René, La dérive des continents. Journal des instituteurs et des institutrices, 74 (36) (1928) 139-140

Perrier, Georges, «L'hypothèse de Wegener trouve-t-elle sa justification dans les théories et les observations de la Géodésie?», Bulletin de la Société géologique France 23 (4) (1923) 264-269

Porter, Roy, «The Industrial Revolution and the Rise of the Science of Geology», dans: Teich, M./R. M. Young (eds.), Changing Perspectives in the History of Science. Essay in Honour of Joseph Needham (London 1973) 320-343

Russo, Philibert, «Recherches sur les déplacements tectoniques des aires continentales», Mémoire de la Société des Sciences naturelles du Maroc 25 (1930)

Russo, Philibert, Les déplacements des continents (Gap 1933)

Scalera, Giancarlo, «Roberto Mantovani (1854-1933) and his ideas on the expanding Earth as revealed by his correspondence and manuscripts», Annals of Geophysics 52 (6) (2009) 615-649

Schaer, Jean-Paul, «Émile Argand 1879-1940. Life and portrait of an inspired geologist», Eclogae Geologicae Helvetiae 84 (3) (1991) 511-534

Snider, Antonio, La création et ses mystères dévoilés (Paris 1859)

Stoyko, Nicolas, «Sur les déplacements périodiques des continents», Comptes rendus hebdomadaires des séances de l'Académie des sciences Paris 94 (1932) 2215-2226

Suess, Eduard, Das Antlitz der Erde (Leipzig 1885-1909)

Termier, Pierre, «Sur la nécessité d'une nouvelle interprétation de la tectonique des Alpes franco-italiennes», Bulletin de la Société géologique de France $4^{\mathrm{e}}$ série 7 (1907) 174-189

Termier, Pierre, «L'Atlantide», Revue scientifique 35 (2) (1913) 33-41

Termier, Pierre, «La dérive des continents», Revue scientifique 62 (9) (1924) 257-267

Termier, Pierre, La joie de connaitre. Souvenirs d'un géologue (Paris 1925)

Termier, Pierre, La vocation de savant. Souvenirs d'un géologue (Paris 1929)

Vincent, Pierre, Sciences naturelles. Classe de quatrième des lycées et collèges (Paris 1953)

Vinot, H., «Une belle page de géographie», Le Madécasse 1240 (1932) 3

Wegener, Alfred Lothar, «Die astronomischen Werke Alfons X.», Bibliotheca mathematica $3^{\mathrm{e}}$ série, 6 (1905) 129-185

Wegener, Alfred Lothar, Thermodynamik der Atmosphäre (Leipzig 1911)

Wegener, Alfred Lothar, Die Entstehung der Kontinente und Ozeane (Braunschweig 1915)

Wegener Alfred Lothar, La genèse des continents et des océans, traduit de la troisième édition allemande par M. Reichel (Paris 1924)

Wegener, Alfred Lothar, La genèse des continents et des océans. Théorie des translations continentales, nouvelle traduction française d'après la cinquième et dernière édition allemande (1928) par Armand Lerner (Paris 1937) (Réédition C. Bourgois, Paris 1990)

Willis, Bailey, «Principles of paleogeography», Science 31 (790) (1910) 241-260

Wood, Robert M., The Dark Side of the Earth (London 1985) 